\title{
Huperzine A: is it an effective disease-modifying drug for Alzheimer's disease?
}

\author{
Zhong Ming Qian ${ }^{1}$ and $\mathrm{Ya} \mathrm{Ke}^{2 *}$ \\ 1 Laboratory of Neuropharmacology, Fudan University School of Pharmacy, Shanghai, China \\ ${ }^{2}$ School of Biomedical Sciences, Faculty of Medicine, The Chinese University of Hong Kong, Shatin, NT, Hong Kong, China
}

\section{Edited by:}

Sofía Díaz Cintra, Universidad

Nacional Autónoma de México,

Mexico

Reviewed by:

Rongqiao He, Chinese Academy of

Sciences, China

Yen-Yu lan Shih, University of North

Carolina at Chapel Hill, USA

*Correspondence:

Ya Ke, School of Biomedical Sciences, Faculty of Medicine, The Chinese

University of Hong Kong, Shatin, NT, Hong Kong, China

e-mail: yake@cuhk.edu.hk
Alzheimer's disease $(A D)$ is a progressive neurodegenerative disorder for which there is no cure. Huperzine A (HupA) is a natural inhibitor of acetylcholinesterase (AChE) derived from the Chinese folk medicine Huperzia serrata (Qian Ceng Ta). It is a licensed anti-AD drug in China and is available as a nutraceutical in the US. A growing body of evidence has demonstrated that HupA has multifaceted pharmacological effects. In addition to the symptomatic, cognitive-enhancing effect via inhibition of AChE, a number of recent studies have reported that this drug has "non-cholinergic" effects on AD. Most important among these is the protective effect of HupA on neurons against amyloid beta-induced oxidative injury and mitochondrial dysfunction as well as via the up-regulation of nerve growth factor and antagonizing $\mathrm{N}$-methyl-D-aspartate receptors. The most recent discovery that HupA may reduce brain iron accumulation lends further support to the argument that HupA could serve as a potential disease-modifying agent for $A D$ and also other neurodegenerative disorders by significantly slowing down the course of neuronal death.

Keywords: huperzine A, Alzheimer's disease, acetylcholinesterase inhibitor, disease-modifying agent, noncholinergic effects

\section{INTRODUCTION}

Huperzine A (HupA) is a natural inhibitor of acetylcholinesterase (AChE) derived from the Chinese folk medicine Huperzia serrata (Qian Ceng Ta) (Ratia et al., 2013). There is a long history of using $H$. serrata as a medicine in China to treat different kinds of disorders, including bruises, strains, swelling, rheumatism, schizophrenia, myasthenia gravis, and fever (Skolnick, 1997; Ma et al., 2007). HupA is a licensed anti-Alzheimer's disease drug in China and is available as a nutraceutical in the US (Orhan et al., 2011). A growing body of evidence has demonstrated that HupA could effectively reverse or attenuate cognitive deficits in rodents, primates, and human (Howes and Perry, 2011). A recent systematic review and meta-analysis of randomized clinical trials concluded that HupA could improve cognitive function, daily living activity, and global clinical assessment in patients with Alzheimer's disease (AD), with relatively few and mild adverse effects mainly related to its effect on the cholinergic system (Xing, 2014; Yang et al., 2014).

Currently, the pharmacological mechanisms of HupA in the treatment of $\mathrm{AD}$ have not been fully detailed. However, a number of recent studies have reported that this drug has "non-cholinergic" effects on AD (Ratia et al., 2013; Huang et al., 2014) in addition to the symptomatic, cognitive-enhancing effect of cholinesterase

Abbreviations: $A \beta$, amyloid- $\beta$ peptide; $A C h E$, acetylcholinesterase; $A D$, Alzheimer's disease; APP, amyloid precursor protein; APP/PS1, APPswe/PS1dE9; ATP, adenosine 5 '-triphosphate; BFCNs, basal forebrain cholinergic neurons; CAT, catalase; GSH$\mathrm{Px}$, glutathione peroxidase; GSK-3, glycogen synthase kinase- 3 ; $\mathrm{H}_{2} \mathrm{O}_{2}$, hydrogen peroxide; HupA, huperzine A; MDA, malondialdehyde; NFTs, neurofibrillary tangles; NGF, nerve growth factor; NMDA, $N$-methyl-D-aspartate; PKC, protein kinase $\mathrm{C}$; ROS, reactive oxygen species; SOD, superoxide dismutase; TBI, transferrin-bound iron; TfR1, transferrin receptor 1; TrkA, tropomyosin kinase receptor A. inhibition (Wang et al., 1986; Liu and Liu, 1995; Zhu and Giacobini, 1995). These new findings have greatly improved our understanding of pharmacological mechanisms of HupA in the treatment of $\mathrm{AD}$. This article focuses on the recent advances in the studies on "non-cholinergic" roles of HupA. The updated understanding of the "cholinergic" action of HupA has recently been well-reviewed elsewhere (Fu and Li, 2011; Howes and Perry, 2011).

\section{SENILE PLAQUES AND NEUROFIBRILLARY TANGLES: CONSEQUENCES RATHER THAN INITIAL EVENTS}

Alzheimer's disease is a progressive neurological disorder clinically characterized by memory loss, mental deterioration, and impairment in the activities of daily living and behavioral disturbances throughout the disease course. The senile plaques and neurofibrillary tangles (NFTs), which are composed of self-polymerized amyloid- $\beta$ peptide $(A \beta)$ and hyperphosphorylated tau proteins, respectively, are the two major pathological hallmarks in $\mathrm{AD}$ brains (Zhao et al., 2009; Maeda et al., 2011). Although much progress has been made, the etiology of AD is still unclear and hence no preventive measure and effective disease-modifying treatment for this disease are currently available (Citron, 2010).

Accumulated data showed that senile plaques and NTFs are associated with the death of cholinergic neurons in AD. However, these two major pathological hallmarks might be just a consequence of the disease process rather than an initial event that causes AD (Huang et al., 2014). The failed result of several major clinical trials targeting $A \beta$ (Ayton et al., 2012) is one of the strongest supports for this viewpoint. Doubtless, further studies on pathophysiological mechanisms involved in the formation of these two major pathological hallmarks in $\mathrm{AD}$ are fundamental 
and critical not only for elucidating the etiology of AD but also for the development of a disease-modifying treatment for AD. Also, further studies on the "non-cholinergic" of HupA might provide important insights into the etiology of AD.

\section{NON-CHOLINERGIC EFFECTS ON ALZHEIMER'S DISEASE}

A number of recent studies have reported that HupA has neuroprotective properties, possessing both "cholinergic" and "noncholinergic" effects on AD. Here, we will discuss the major non-cholinergic effects of HupA on AD.

\section{PROTECTING NEURONS AGAINST A $\beta$-INDUCED OXIDATIVE INJURY AND APOPTOSIS}

Increased oxidative stress is associated with a number of neurodegenerative diseases, including $\mathrm{AD}$. It has been well documented that $\mathrm{A} \beta$ treatment can generate oxidative stress that eventually triggers a state of neurotoxicity and cell death (Xiao et al., 2002). Studies have demonstrated that HupA could enhance the cell viability and the activities of antioxidant enzymes including glutathione peroxidase (GSH-Px), superoxide dismutase (SOD), and catalase (CAT); decrease the level of malondialdehyde (MDA) in PC12 (neuron-like rat pheochromocytoma) cells and cultured rat primary cortical neurons (Xiao et al., 2000a,b); and markedly reduced the MDA level in chronic cerebral hypo-perfusion rats (Wang et al., 2000) and aged rats (Shang et al., 1999). The protective effect of HupA on $\mathrm{A} \beta$-induced cell lesion was also observed in NG108-15 cells (Zhang et al., 2002a). These results indicate that HupA can function as an antioxidant in $\mathrm{A} \beta$-induced oxidative stress model by increasing the activities of antioxidant enzymes.

The neuroprotective effects of HupA against $A \beta$-induced oxidative injury are at least partly associated with its anti-apoptotic action. There is considerable evidence showing that $\mathrm{A} \beta$ can activate intracellular apoptosis pathways, which lead to neuronal death (Saille et al., 1999). Activation of caspase-3 is a key event in the execution of apoptotic cascade in central nervous system diseases. It has been demonstrated that HupA could attenuate the increase of caspase- 3 activity induced by $A \beta$ in cultured primary cortical neurons (Xiao et al., 2002) and inhibit $A \beta$-induced apoptosis by reversing the down-regulation of the expression of $\mathrm{Bcl}-2$ and the up-regulation of the expressions of Bax and p53 (Wang et al., 2001a). HupA had the same effects on $\mathrm{H}_{2} \mathrm{O}_{2}$-induced apoptosis with a down-regulation of the bax and p53 genes and up-regulation of the Bcl-2 gene to normal levels (Wang et al., 2001b).

\section{AMELIORATING MITOCHONDRIAL MALFUNCTION IN ALZHEIMER'S DISEASE BRAIN}

Perturbations in mitochondrial function have long been observed in samples derived from clinically confirmed patients with $\mathrm{AD}$, including altered mitochondrial morphology, compromised enzyme complexes in the tricarboxylic acid cycle, and reduced cytochrome $c$ oxidase activity (Cardoso et al., 2001). In addition, accumulated evidence showed that mitochondria are direct targets of $A \beta$ (Yao et al., 2011). A $\beta$ could be accumulated within mitochondria and interact with a mitochondrial protein, A $\beta$ bindingalcohol-dehydrogenase, resulting in decreased cytochrome $c$ oxidase activity and increased oxidative stress (Reddy and Beal, 2008).
The neurotoxicity induced by $\mathrm{A} \beta$ will trigger a vicious cycle in which excessive $A \beta$ accumulation and sustained mitochondrial dysfunction synergize to activate a cascade of neurodegenerative pathways (Silva et al., 2012).

Studies have provided evidence that HupA has the ability to effectively ameliorate the mitochondrial malfunction. $A \beta(25-$ 35 ) treatment could lead to a rapid decline of adenosine 5'triphosphate (ATP) level, an obvious disruption of mitochondrial membrane homeostasis and integrity, a reduction in key enzyme activities in the electron transport chain and the tricarboxylic acid cycle, and an increase in intracellular reactive oxygen species (ROS) in PC12 cells, while HupA not only attenuated these signs of cellular stress caused by A $\beta$, but also enhanced ATP concentration and decreased ROS accumulation (Gao and Tang, 2006). In isolated rat brain mitochondria, HupA was able to effectively prevent $A \beta$-induced mitochondrial swelling, ROS increase, and cytochrome $c$ release. Furthermore, it could also ameliorate $A \beta$ induced decreases in mitochondrial respiration, ATP synthesis, mitochondrial respiratory chain enzyme activity, and transmembrane potential (Gao et al., 2009). There is no evidence for the existence of cholinergic system in isolated brain mitochondria. Therefore, the mitochondria-targeted effects of HupA are clearly independent of cholinergic system. In addition, HupA could inhibit the penetration of $A \beta$ into mitochondria and ameliorated $A \beta$-induced dysfunction of tricarboxylic acid cycle in isolated brain cortical mitochondria (Yang et al., 2012).

\section{ANTAGONIZING EFFECTS ON $\boldsymbol{N}$-METHYL-D-ASPARTATE RECEPTORS}

Synaptic plasticity, the variable efficacy of neurotransmission at synapses, is thought to underlie the ability of the brain to store memories (Martin et al., 2000). The forms of synaptic plasticity that are most likely to be involved in memory storage require gene transcription and protein synthesis to stabilize synaptic changes over time (Adams and Dude, 2005). Glutamatergic synapses mediate virtually all excitatory neurotransmission in mammalian brains (Sucher et al., 1996). Glutamate released from presynaptic terminals activates several types of glutamate-gated ion channels on postsynaptic membranes, including $N$-methyl-D-aspartate (NMDA) receptors (Rao and Finkbeiner, 2007). Permeability to $\mathrm{Ca}^{2+}$ is a feature of all NMDA receptors (NMDA-R), which are composed of an essential NR1 subunit and multiple NR2 subunits (Rao and Finkbeiner, 2007). Excitotoxicity caused by disturbances of glutamatergic neurotransmission in the brain has been shown to be involved in the pathogenesis of AD (Emilien et al., 2000). NMDA receptor antagonists have been used as neuroprotective agents to ameliorate the cognition deficits of patients with $\mathrm{AD}$ (Emilien et al., 2000; Marx, 2000).

Huperzine A could inhibit NMDA-induced toxicity in a dosedependent way in cultured primary neuronal cells (Marx, 2000). HupA interacts with the NMDA ion channel by inhibition of $\left[{ }^{3} \mathrm{H}\right] \mathrm{MK}-801$ and $\left[{ }^{3} \mathrm{H}\right] \mathrm{TCP}$ binding in brain synaptosomal plasma membranes but not the glycine or NMDA ligand-specific sites. The non-competitive binding results suggest that HupA inhibits the NMDA-induced toxicity most likely by blocking NMDA ion channels and the subsequent $\mathrm{Ca}^{2+}$ mobilization at or near the PCP and MK-801 ligand sites (Gordon et al., 2001). HupA reversibly inhibited NMDA-induced current in acutely 
dissociated rat hippocampal pyramidal neurons and blocked specific $\left[{ }^{3} \mathrm{H}\right] \mathrm{MK}-801$ binding in synaptic membranes from rat cerebral cortex (Wang et al., 1999) and the inhibitory effect is noncompetitive (Zhang and $\mathrm{Hu}, 2001$ ). HupA acts as antagonist of the NMDA-R, acting at one of the polyamine binding sites on the NMDA-R (Zhang et al., 2002b).

\section{REGULATING THE EXPRESSION AND SECRETION OF NERVE GROWTH FACTOR}

Nerve growth factor (NGF), a neurotrophin, plays a trophic role both during development and in adulthood (Aloe et al., 2012), and exerts its biological action by interacting with the specific receptor tropomyosin kinase receptor A (TrkA) (Huang and Reichard, 2003). Studies on rodents (Fischer et al., 1987) and primates (Tuszynski et al., 1991) have demonstrated that exogenous NGF was able to protect basal forebrain cholinergic neurons (BFCNs) from both traumatic insults and age-related cholinergic decline. Also, NGF is able to regulate both amyloid gene expression and protein processing (Rossner et al., 1998) and to counteract tau hyperphosphorylation (Zhang et al., 2010), acting directly at the two classical hallmarks of AD. A decreased NGF immunoreactivity in the BFCNs of patients with AD suggested that impaired NGF supply via retrograde transport could be the effective cause of cholinergic neurodegeneration in AD (Scott et al., 1995). These findings plus the experimental studies on NGF deficit-induced neurodegeneration in transgenic mice demonstrated a novel causal link between neurotrophic signaling deficits and Alzheimer's neurodegeneration (Cattaneo and Calissano, 2012). Alteration in the homeostasis and equilibrium of NGF processing and NGF/TrkA signaling in target neurons has been considered as an upstream driver of all the cellular and molecular central hallmarks of AD (Tang et al., 2005).

Therefore, it will be very important to re-establish a correct homeostatic balance between ligands, and receptors, of the NGF pathway (Tang et al., 2005). Wang et al. (2006) investigated the effects of HupA on secretion of NGF in cultured rat cortical astrocytes and neurite outgrowth in rat PC12 cells and demonstrated that HupA treatment induced a significant increase in both mRNA and protein levels of NGF in astrocytes. They also found that treatment of PC12 cells with HupA led to a significant increase in the number of neurite-bearing cells without significant alteration in cell viability or other signs of cytotoxicity. The positive effect of HupA on the mRNA and protein levels of NGF, accompanied by the inhibitory effects on the memory deficits and neuronal damage, have also been reported in mice treated with transient cerebral ischemia and re-perfusion (Wang et al., 2006). These findings suggested that HupA has a direct or indirect neurotrophic activity, which might be beneficial in treatment of neurodegenerative disorders such as AD (Tang et al., 2005).

\section{PROMOTING NON-AMYLOIDOGENIC AMYLOID PRECURSOR PROTEIN PROCESSING BY ACTIVATING PROTEIN KINASE C AND THE Wnt/更-CATENIN SIGNALING PATHWAY}

The canonical Wnt signaling pathway has an important role in development and maintenance of the nervous system and is also associated with neurodegenerative diseases (De Ferrari et al., 2003). Loss of Wnt signaling function is involved in $A \beta$-dependent neurodegeneration in the $\mathrm{AD}$ brain and two key components of the canonical Wnt signaling pathway, glycogen synthase kinase (GSK)-3 $\beta$ and $\beta$-catenin, are altered in the AD model mouse brain (Pei et al., 1999). It has also been reported that activation of Wnt signaling can prevent neurodegeneration induced by $\mathrm{A} \beta$ fibrils (De Ferrari et al., 2003) and that inhibition of GSK-3 $\beta$ and enhancement of $\beta$-catenin activity could prevent the loss of function of the Wnt signaling pathway caused by A $\beta$ toxicity (Farias et al., 2005).

Wang et al. (2011) showed that HupA has a role in the regulation of Wnt signaling in APPswe/PS1dE9 (APP/PS1) transgenic mouse and APPsw cell models and could significantly inhibit GSK$3 \beta$ activity and stabilize $\beta$-catenin protein level in vivo and in vitro. They also found that HupA induced a significant increase in the phosphorylation levels of both GSK-3 $\alpha$ and GSK-3 $\beta$ proteins in APP/PS1 mouse brain and APPsw-overexpressing cells, while activation of both GSK- $3 \alpha$ and GSK- $3 \beta$ through their autophosphorylation has been implicated in $\mathrm{AD}$ pathogenesis (Caricasole et al., 2004). Their findings suggested that HupA can inhibit the activity of GSK-3 $\alpha / \beta$ and, hence, may inhibit $A \beta$ generation and tau phosphorylation. HupA reverses protein kinase $C$ (PKC)- and Wnt-inhibitor-induced inhibition of non-amyloidogenic processing of amyloid precursor protein (APP), paralleled by an inactivation of GSK-3 and a reversal of the level of $\beta$-catenin (Zhang et al., 2004; Wang et al., 2011). These findings imply that HupA is involved in regulation of the Wnt signaling pathway and that HupA regulates APP processing to the non-amyloidogenic pathway at least partly through activation of $\mathrm{PKC}$ and $\mathrm{Wnt} / \beta$-catenin signaling.

\section{REDUCING IRON IN AD BRAIN}

Misregulation in brain iron has been considered to be one of the primary causes of neuronal death in neurodegenerative disorders (Qian et al., 1997; Qian and Shen, 2001; Lei et al., 2012). Evidence has also been gathered to imply that $\mathrm{A} \beta$ production, precipitation, and toxicity in $\mathrm{AD}$ are caused by abnormal interactions with neocortical iron (Zhu et al., 2004; Zhao et al., 2008; Smith et al., 2010). In a recent study (Huang et al., 2014), we demonstrated for the first time that HupA was able to reduce significantly the contents of insoluble and soluble $A(\beta-40$ and $A(\beta-42$ and hyperphosphorylated tau in the brain of APP/PS1 transgenic mice. Also, HupA could decrease the deposition of amyloid plaques and the levels of oligomeric A $\beta$. It also suppressed APP695 expression and increase ADAM10 contents in APP/PS1 mice brain. However, all of these beneficial effects of HupA could be largely abolished by high iron diet. In addition, the iron levels in the hippocampus and cortex of the APP/PS1 mice were found to be elevated while quantitative analysis revealed that the abnormal increase in brain iron contents in these two regions were almost completely normalized by the chronic treatment with HupA. Our findings provided direct evidence for the inhibitory effect of HupA on brain iron, and implied that the beneficial effects of HupA on AD is caused by the reduction in brain iron in the APP/PS1 mice.

Furthermore, we showed that mutation of APPswe/PS1dE9 could lead to a significant increase in transferrin receptor 1 (TfR1) expression and that HupA was able to induce a significant reduction in TfR1 expression in the brain of APP/PS1 mice in vivo, and a remarkable reduction in TfR1 expression as well 
as transferrin-bound iron (TBI) uptake in the cultured neurons in vitro. Two AChE inhibitors donepezil or galantamine did not induce any changes in TfR1 expression in the brain and had no effect on TBI uptake by the neurons, indicating that effects of HupA on TfR1 expression and TBI uptake were independent of its anti-AChE action. Based on the above findings, we propose that HupA has the ability to inhibit TfR 1 expression and then reduce TBI uptake by the neurons or other brain cells, leading to a progressive reduction in iron contents and also iron-induced oxidative stress in the brain (Huang et al., 2014).

\section{DISEASE-MODIFYING POTENTIAL OF HUPERZINE A}

Currently Hup A is proved to be used in China to improve symptoms of $\mathrm{AD}$ (Zangara, 2003). The information available from clinical trials of Hup A was mainly on patients with mild and moderate $\mathrm{AD}$, showing significant improvement in their cognitive functions (Rafii et al., 2011; Yang et al., 2014). However, due to the relatively short study duration ( $<6$ months) and lack of other pathological assessments, it is still premature to conclude from these trials that HupA could slow down the progress of AD. On the other hand, studies on animal model of AD (e.g., APP/PS1 mice) have shown that HupA could suppress $\mathrm{A} \beta$ accumulation, and amyloid plaques and hyperphosphorylated tau formation when drug administration began at an early stage (Wang et al., 2011; Huang et al., 2014). This result implied that HupA may be also beneficial if taken at preclinical stage of $\mathrm{AD}$. Therefore, further long-term longitudinal clinical trials and preclinical studies are demanded in determining the optimal time to start the treatment and monitoring of disease progression. Additional studies will also be needed to address the safety of long-term treatment.

\section{SUMMARY}

Studies on non-cholinergic roles of HupA have made important contributions for the understanding of pharmacological mechanisms of HupA in the treatment of $\mathrm{AD}$. In addition to the symptomatic, cognitive-enhancing effect via cholinesterase inhibition, HupA has a number of "non-cholinergic" effects on AD, including the ability to protect neurons against $A \beta$-induced oxidative injury and apoptosis, to ameliorate mitochondrial malfunction, to antagonize NMDA-R, to regulate NGF, promote nonamyloidogenic APP processing, and to reduce iron in the brain (Figure 1). Therefore, this multiplicity of action renders HupA a

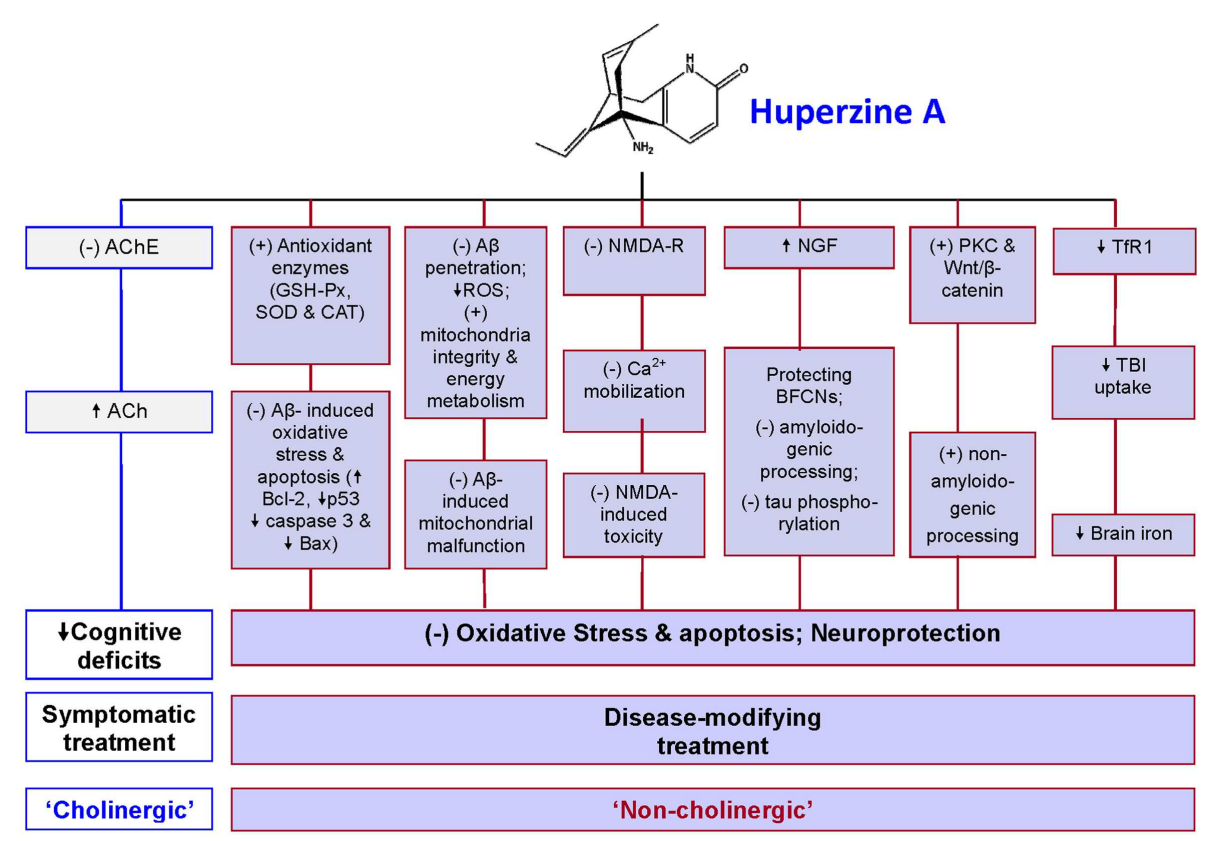

FIGURE 1 | A summary of pharmacological mechanisms of huperzine $A$ (HupA) in the treatment of Alzheimer's disease (AD). In addition to acting as an acetylcholinesterase (AchE) inhibitor, HupA has non-cholinergic roles in the treatment of $A D$ to protect neurons and other brain cells from oxidative stress damage and apoptosis. It has been demonstrated that HupA has the ability: (1) to protect neurons against $A \beta$-induced oxidative injury and apoptosis by enhancing the activities of antioxidant enzymes including glutathione peroxidase (GSH-Px), superoxide dismutase (SOD), and catalase (CAT), attenuating the $A \beta$-induced increase in caspase- 3 activity, and inhibiting $A \beta$-induced apoptosis by reversing the down-regulation of the expression of $\mathrm{Bcl}-2$ and the up-regulation of $\mathrm{Bax}$ and p53 expressions; (2) to ameliorate mitochondrial malfunction in $A D$ brain by preventing $A \beta$-penetration into the mitochondria, suppressing ROS production, and improving mitochondrial integrity and energy metabolism, thus minimizing $A \beta$-induced mitochondrial malfunction; (3) to act as antagonist of the NMDA receptors (NMDA-R) to inhibit the NMDA-induced toxicity by blocking NMDA ion channels and the subsequent $\mathrm{Ca}^{2+}$ mobilization; (4) to increase nerve growth factor (NGF), which protects basal forebrain cholinergic neurons (BFCNs) from both traumatic insults and age-related cholinergic decline and regulate both amyloid gene expression and protein processing and counteract tau hyperphosphorylation, acting directly at the two classical hallmarks of $\mathrm{AD}$; (5) to promote non-amyloidogenic processing by activating protein kinase $C$ (PKC) and the $\mathrm{Wnt} / \beta$-catenin signaling pathway; and (6) to inhibit transferrin receptor 1 (TfR1) expression and then reduce transferrin-bound iron (TBI) uptake by the neurons or other brain cells, which have TfR1 expression on the membrane, leading to a progressive reduction in iron contents and also iron-induced oxidative stress in the brain. Based on these roles, it is reasonable to consider HupA as an effective disease-modifying drug for $A D .(+)=$ stimulate; $(-)=$ inhibit; $\uparrow=$ increase; $\downarrow=$ decrease; Ach = acetylcholine. 
highly effective drug and potentially it could serve as a diseasemodifying drug for AD. It may also be used for the treatment of other neurodegenerative disorders although further studies are needed.

\section{AUTHOR CONTRIBUTIONS}

Ya Ke and Zhong Ming Qian analyzed the data and wrote the paper.

\section{ACKNOWLEDGMENTS}

The studies in our laboratories were supported by the Competitive Earmarked Grants of The Hong Kong Research Grants Council (GRF 466713), National 973 Programs (2011CB510004, 2014CB541604), the General Grant of National Natural Science Foundation of China (NSFC) (31271132, 31371092), and Key Project Grant of NSFC (31330035).

\section{REFERENCES}

Adams, J. P., and Dude, S. M. (2005). Late-phase long-term potentiation: getting to the nucleus. Nat. Rev. Neurosci. 6, 737-743. doi:10.1038/nrn1749

Aloe, L., Rocco, M. L., Branching, P., and Mani, L. (2012). Nerve growth factor: from the early discoveries to the potential clinical use. J. Transl. Med. 10, 239. doi:10.1186/1479-5876-10-239

Ayton, S., Lei, P., and Bush, A. I. (2012). Homeostasis in Alzheimer's disease. Free Radic. Biol. Med. 62, 76-89. doi:10.1016/j.freeradbiomed.2012.10.558

Cardoso, S. M., Santos, S., Swerdlow, R. H., and Oliveira, C. R. (2001). Functional mitochondria are required for amyloid beta-mediated neurotoxicity. FASEB J. $15,1439-1441$.

Caricasole, A., Copani, A., Caraci, F., Aronica, E., Rozemuller, A. J., Caruso, A., et al. (2004). Induction of Dickkopf-1, a negative modulator of the Wnt pathway, is associated with neuronal degeneration in Alzheimer's brain. J. Neurosci. 24, 6021-6027. doi:10.1523/JNEUROSCI.1381-04.2004

Cattaneo, A., and Calissano, P. (2012). Nerve growth factor and Alzheimer's disease: new facts for an old hypothesis. Mol. Neurobiol. 46, 588-604. doi:10.1007/ s12035-012-8310-9

Citron, M. (2010). Alzheimer's disease: strategies for disease modification. Nat. Rev. Drug Discov. 9, 387-398. doi:10.1038/nrd2896

De Ferrari, G. V., Chacon, M. A., Barria, M. I., Garrido, J. L., Godoy, J. A., Olivares, G., et al. (2003). Activation of Wnt signaling rescues neurodegeneration and behavioral impairments induced by beta-amyloid fibrils. Mol. Psychiatry 8, 195-208. doi:10.1038/sj.mp.4001208

Emilien, G., Beyreuther, K., Masters, C. L., and Maloteaux, J. M. (2000). Prospects for pharmacological intervention in Alzheimer disease. Arch. Neurol. 57, 454-459. doi:10.1001/archneur.57.4.454

Farias, G. G., Godoy, J. A., Vazquez, M. C., Adani, R., Meshulam, H., Avila, J., et al. (2005). The anti-inflammatory and cholinesterase inhibitor bifunctional compound IBU-PO protects from beta-amyloid neurotoxicity by acting on Wnt signaling components. Neurobiol. Dis. 18, 176-183. doi:10.1016/j.nbd.2004.09.012

Fischer, W., Wictorin, K., Bjorklund, A., Williams, L. R., Varon, S., and Gage, F. H. (1987). Amelioration of cholinergic neuron atrophy and spatial memory impairment in aged rats by nerve growth factor. Nature 329, 65-68. doi:10.1038/329065a0

Fu, L. M., and Li, J. T. (2011). A systematic review of single Chinese herbs for Alzheimer's disease treatment. Evid. Based Complement. Alternat. Med. 2011, 640284. doi:10.1093/ecam/nep136

Gao, X., and Tang, X. C. (2006). Huperzine A attenuates mitochondrial dysfunction in beta-amyloid-treated PC12 cells by reducing oxygen free radicals accumulation and improving mitochondrial energy metabolism. J. Neurosci. Res. 83, 1048-1057. doi:10.1002/jnr.20791

Gao, X., Zheng, C. Y., Yang, L., Tang, X. C., and Zhang, H. Y. (2009). Huperzine A protects isolated rat brain mitochondria against beta-amyloid peptide. Free Radic. Biol. Med. 46, 1454-1462. doi:10.1016/j.freeradbiomed.2009.02.028

Gordon, R. K., Nigam, S. V., Weitz, J. A., Dave, J. R., Doctor, B. P., and Ved, H. S. (2001). The NMDA receptor ion channel: a site for binding of huperzine A. J. Appl. Toxicol. 21(Suppl. 1), S47-S51. doi:10.1002/jat.805
Howes, M. J., and Perry, E. (2011). The role of phytochemicals in the treatment and prevention of dementia. Drugs Aging 28, 439-468. doi:10.2165/11591310000000000-00000

Huang, E. J., and Reichard, L. F. (2003). TRK receptors: roles in neuronal signal transduction. Annu. Rev. Biochem. 72, 609-642. doi:10.1146/annurev.biochem. 72.121801 .161629

Huang, X. T., Qian, Z. M., He, X., Gong, Q., Wu, K. C., Jiang, L. R., et al. (2014). Reducing iron in the brain: a novel pharmacologic mechanism of huperzine A in the treatment of Alzheimer's disease. Neurobiol. Aging 35, 1045-1054. doi:10.1016/j.neurobiolaging.2013.11.004

Lei, P., Ayton, S., Finkelstein, D. I., Spoerri, L., Ciccotosto, G. D., Wright, D. K., et al. (2012). Tau deficiency induces parkinsonism with dementia by impairing APP-mediated iron export. Nat. Med. 18, 291-295. doi:10.1038/nm.2613

Liu, M. Y., and Liu, H. C. (1995). Intelligence promoting Chinese materia medica. [Article in Chinese]. Zhongguo Zhong Xi Yi Jie He Za Zhi 15, 59-61.

Ma, X., Tan, C., Zhu, D., Gang, D. R., and Xiao, P. (2007). Huperzine A from Huperzia species - an ethnopharmacolgical review. J. Ethnopharmacol. 113, 15-34. doi:10.1016/j.jep.2007.05.030

Maeda, J., Zhang, M. R., Okauchi, T., Ji, B., Ono, M., Hattori, S., et al. (2011). In vivo positron emission tomographic imaging of glial responses to amyloid-beta and tau pathologies in mouse models of Alzheimer's disease and related disorders. J. Neurosci. 31, 4720-4730. doi:10.1523/JNEUROSCI.3076-10.2011

Martin, S. J., Grimwood, P. D., and Morris, R. G. (2000). Synaptic plasticity and memory: an evaluation of the hypothesis. Annu. Rev. Neurosci. 23, 649-711. doi:10.1146/annurev.neuro.23.1.649

Marx, J. (2000). Alzheimer's congress. Drug shows promise for advanced disease. Science 289, 375-377. doi:10.1126/science.289.5478.375b

Orhan, I. E., Orhan, G., and Gurkas, E. (2011). An overview on natural cholinesterase inhibitors - a multi-targeted drug class - and their mass production. Mini Rev. Med. Chem. 11, 836-842. doi:10.2174/138955711796575434

Pei, J. J., Braak, E., Braak, H., Grundke-Iqbal, I., Iqbal, K., Winblad, B., et al. (1999). Distribution of active glycogen synthase kinase 3beta (GSK-3beta) in brains staged for Alzheimer disease neurofibrillary changes. J. Neuropathol. Exp. Neurol. 58, 1010-1019. doi:10.1097/00005072-199909000-00011

Qian, Z. M., and Shen, X. (2001). Brain iron transport and neurodegeneration. Trends Mol. Med. 7, 103-110. doi:10.1016/S1471-4914(00)01910-9

Qian, Z. M., Wang, Q., and Pu, Y. (1997). Brain iron and neurological disorders. Chin. Med. J. 110, 455-458.

Rafii, M. S., Walsh, S., Little, J. T., Behan, K., Reynolds, B., Ward, C., et al. (2011). A phase II trial of huperzine A in mild to moderate Alzheimer disease. Neurology 76, 1389-1394. doi:10.1212/WNL.0b013e318216eb7b

Rao, V. R., and Finkbeiner, S. (2007). NMDA and AMPA receptors: old channels, new tricks. Trends Neurosci. 30, 284-291. doi:10.1016/j.tins.2007.03.012

Ratia, M., Giménez-Llort, L., Camps, P., Muñoz-Torrero, D., Pérez, B., Clos, M. V., et al. (2013). Huprine $\mathrm{X}$ and huperzine $\mathrm{A}$ improve cognition and regulate some neurochemical processes related with Alzheimer's disease in triple transgenic mice (3xTg-AD). Neurodegener. Dis. 11, 129-140. doi:10.1159/000336427

Reddy, P. H., and Beal, M. F. (2008). Amyloid beta, mitochondrial dysfunction and synaptic damage: implications for cognitive decline in aging and Alzheimer's disease. Trends Mol. Med. 14, 45-53. doi:10.1016/j.molmed.2007.12.002

Rossner, S., Ueberham, U., Schliebs, R., Perez-Polo, J. R., and Bigl, V. (1998). The regulation of amyloid precursor protein metabolism by cholinergic mechanisms and neurotrophin receptor signaling. Prog. Neurobiol. 56, 541-569. doi:10.1016/S0301-0082(98)00044-6

Saille, C., Marin, P., Martinou, J. C., Nicole, A., London, J., and Ceballos-Picot, I. (1999). Transgenic murine cortical neurons expressing human Bcl-2 exhibit increased resistance to amyloid beta-peptide neurotoxicity. Neuroscience 92, 1455-1463. doi:10.1016/S0306-4522(99)00089-5

Scott, S. A., Mufson, E. J., Weingartner, J. A., Skau, K. A., and Crutcher, K. A. (1995). Nerve growth factor in Alzheimer's disease: increased levels throughout the brain coupled with declines in nucleus basalis. J. Neurosci. 15, 6213-6221.

Shang, Y. Z., Ye, J. W., and Tang, X. C. (1999). Improving effects of huperzine A on abnormal lipid peroxidation and superoxide dismutase in aged rats. Zhongguo Yao Li Xue Bao 20, 824-828.

Silva, D. F., Selfridge, J. E., Lu, J. H., Lezi, E., Cardoso, S. M., and Swerdlow, R. H. (2012). Mitochondrial abnormalities in Alzheimer's disease: possible targets for therapeutic intervention. Adv. Pharmacol. 64, 83-126. doi:10.1016/B978-0-12394816-8.00003-9 
Skolnick, A. A. (1997). Old Chinese herbal medicine used for fever yields possible new Alzheimer disease therapy. JAMA 277, 776. doi:10.1001/jama.1997. 03540340010004

Smith, M. A., Zhu, X., Tabaton, M., Liu, G., McKeel, D. W. Jr., Cohen, M. L., et al. (2010). Increased iron and free radical generation in preclinical Alzheimer disease and mild cognitive impairment. J. Alzheimers Dis. 19, 363-372. doi:10.3233/ JAD-2010-1239

Sucher, N. J., Awobuluyi, M., Choi, Y. B., and Lipton, S. A. (1996). NMDA receptors: from genes to channels. Trends Pharmacol. Sci. 17, 348-355. doi:10.1016/S01656147(96)10046-8

Tang, L. L., Wang, R., and Tang, X. C. (2005). Effects of huperzine A on secretion of nerve growth factor in cultured rat cortical astrocytes and neurite outgrowth in rat PC12 cells. Acta Pharmacol. Sin. 26, 673-678. doi:10.1111/j.1745-7254.2005. 00130.x

Tuszynski, M. H., Sang, H., Yoshida, K., and Gage, F. H. (1991). Recombinant human nerve growth factor infusions prevent cholinergic neuronal degeneration in the adult primate brain. Ann. Neurol. 30, 625-636. doi:10.1002/ana.410300502

Wang, C. Y., Zheng, W., Wang, T., Xie, J. W., Wang, S. L., Zhao, B. L., et al. (2011). Huperzine A activates Wnt/ $\beta$-catenin signaling and enhances the nonamyloidogenic pathway in an Alzheimer transgenic mouse model. Neuropsychopharmacology 36, 1073-1089. doi:10.1038/npp.2010.245

Wang, L. M., Han, Y. F., and Tang, X. C. (2000). Huperzine A improves cognitive deficits caused by chronic cerebral hypoperfusion in rats. Eur. J. Pharmacol. 398, 65-72. doi:10.1016/S0014-2999(00)00291-0

Wang, R., Xiao, X. Q., and Tang, X. C. (2001a). Huperzine A attenuates hydrogen peroxide-induced apoptosis by regulating expression of apoptosis-related genes in rat PC12 cells. Neuroreport 12, 2629-2634. doi:10.1097/00001756-20010828000009

Wang, R., Zhang, H. Y., and Tang, X. C. (2001b). Huperzine A attenuates cognitive dysfunction and neuronal degeneration caused by beta-amyloid protein-(1-40) in rat. Eur. J. Pharmacol. 421, 149-156. doi:10.1016/S0014-2999(01)01030-5

Wang, X. D., Zhang, J. M., Yang, H. H., and Hu, G. Y. (1999). Modulation of NMDA receptor by huperzine A in rat cerebral cortex. Acta Pharmacol. Sin. 20, 31-35.

Wang, Y. E., Yue, D. X., and Tang, X. C. (1986). Anti-cholinesterase activity of huperzine A. [Article in Chinese]. Zhongguo Yao Li Xue Bao 7, 110-113.

Wang, Z. F., Tang, L. L., Yan, H., Wang, Y. J., and Tang, X. C. (2006). Effects of huperzine A on memory deficits and neurotrophic factors production after in mice. Pharmacol. Biochem. Behav. 83, 603-611. doi:10.1016/j.pbb.2006.03.027

Xiao, X. Q., Wang, R., Han, Y. F., and Tang, X. C. (2000a). Protective effects of huperzine A on beta-amyloid(25-35) induced oxidative injury in rat pheochromocytoma cells. Neurosci. Lett. 286, 155-158. doi:10.1016/S0304-3940(00) 01088-0

Xiao, X. Q., Wang, R., and Tang, X. C. (2000b). Huperzine A and tacrine attenuate beta-amyloid peptide-induced oxidative injury. J. Neurosci. Res. 61, 564-569. doi:10.1002/1097-4547(20000901)61:5<564::AID-JNR11>3.3.CO;2-O

Xiao, X. Q., Zhang, H. Y., and Tang, X. C. (2002). Huperzine A attenuates amyloid beta-peptide fragment 25-35-induced apoptosis in rat cortical neurons via inhibiting reactive oxygen species formation and caspase-3 activation. J. Neurosci. Res. 67, 30-36. doi:10.1002/jnr.10075

Xing, S. H., Zhu, C. X., Zhang, R., and An, L. (2014). Huperzine a in the treatment of Alzheimer's disease and vascular dementia: a meta-analysis. Evid. Based Complement Alternat. Med. 2014, 363985. doi:10.1155/2014/363985

Yang, G., Wang, Y., Tian, J., and Liu, J. P. (2014). Huperzine a for Alzheimer's disease: a systematic review and meta-analysis of randomized clinical trials. PLoS ONE 8:e74916. doi:10.1371/journal.pone.0074916

Yang, L., Ye, C. Y., Huang, X. T., Tang, X. C., and Zhang, H. Y. (2012). Decreased accumulation of subcellular amyloid-beta with improved mitochondrial function mediates the neuroprotective effect of huperzine A. J. Alzheimers Dis. 31, 131-142. doi:10.3233/JAD-2012-120274
Yao, J., Rettberg, J. R., Klosinski, L. P., Cadenas, E., and Brinton, R. D. (2011). Shift in brain metabolism in late onset Alzheimer's disease: implications for biomarkers and therapeutic interventions. Mol. Aspects Med. 32, 247-257. doi:10.1016/j.mam.2011.10.005

Zangara, A. (2003). The psychopharmacology of huperzine A: an alkaloid with cognitive enhancing and neuroprotective properties of interest in the treatment of Alzheimer's disease. Pharmacol. Biochem. Behav. 75, 675-686. doi:10.1016/ S0091-3057(03)00111-4

Zhang, H. Y., Liang, Y. Q., Tang, X. C., He, X. C., and Bai, D. L. (2002a). Stereoselectivities of enantiomers of huperzine $\mathrm{A}$ in protection against amyloid 25-35induced injury in PC12 and NG108-15 cells and cholinesterase inhibition in mice. Neurosci. Lett. 317, 143-146. doi:10.1016/S0304-3940(01)02437-5

Zhang, Y. H., Zhao, X. Y., Chen, X. Q., Wang, Y., Yang, H. H., and Hu, G. Y. (2002b). Spermidine antagonizes the inhibitory effect of huperzine A on $[3 \mathrm{H}]$ dizocilpine (MK-801) binding in synaptic membrane of rat cerebral cortex. Neurosci. Lett. 319, 107-110. doi:10.1016/S0304-3940(01)02565-4

Zhang, H. Y., Yan, H., and Tang, X. C. (2004). Huperzine A enhances the level of secretory amyloid precursor protein and protein kinase $\mathrm{C}$-alpha in intracerebroventricular beta-amyloid-(1-40) infused rats and human embryonic kidney 293 Swedish mutant cells. Neurosci. Lett. 360, 21-24. doi:10.1016/j.neulet. 2004. 01.055

Zhang, J. M., and Hu, G. Y. (2001). Huperzine A, a nootropic alkaloid, inhibits Nmethyl-D-aspartate-induced current in rat dissociated hippocampal neurons. Neuroscience 105, 663-669. doi:10.1016/S0306-4522(01)00206-8

Zhang, Z. H., Xi, G. M., Li, W. C., Ling, H. Y., Qu, P., and Fang, X. B. (2010). Cyclic-AMP response element binding protein and tau are involved in the neuroprotective mechanisms of nerve growth factor during focal cerebral ischemia/reperfusion in rats. J. Clin. Neurosci. 17, 353-356. doi:10.1016/j.jocn. 2009.07.086

Zhao, L., Qian, Z. M., Zhang, C., Wing, H. Y., Du, F., and Ya, K. (2008). Amyloid betapeptide 31-35-induced neuronal apoptosis is mediated by caspase-dependent pathways via cAMP-dependent protein kinase A activation. Aging Cell 7, 47-57. doi:10.1111/j.1474-9726.2007.00352.x

Zhao, L., Zhao, S. T., Qian, Z. M., Zhang, C., Wu, X. M., Du, F., et al. (2009). Activation of group III metabotropic glutamate receptor reduces intracellular calcium in beta-amyloid peptide [31-35]-treated cortical neurons. Neurotox. Res. 16, 174-183. doi:10.1007/s12640-009-9068-3

Zhu, X., Raina, A. K., Perry, G., and Smith, M. A. (2004). Alzheimer's disease: the twohit hypothesis. Lancet Neurol. 3, 219-226. doi:10.1016/S1474-4422(04)00707-0

Zhu, X. D., and Giacobini, E. (1995). Second generation cholinesterase inhibitors: effect of (L)-huperzine-A on cortical biogenic amines. J. Neurosci. Res. 41, 828-835. doi:10.1002/jnr.490410613

Conflict of Interest Statement: The authors declare that the research was conducted in the absence of any commercial or financial relationships that could be construed as a potential conflict of interest.

Received: 08 June 2014; accepted: 04 August 2014; published online: 19 August 2014. Citation: Qian ZM and Ke Y (2014) Huperzine A: is it an effective diseasemodifying drug for Alzheimer's disease? Front. Aging Neurosci. 6:216. doi: 10.3389/fnagi.2014.00216

This article was submitted to the journal Frontiers in Aging Neuroscience.

Copyright (C) 2014 Qian and Ke. This is an open-access article distributed under the terms of the Creative Commons Attribution License (CC BY). The use, distribution or reproduction in other forums is permitted, provided the original author(s) or licensor are credited and that the original publication in this journal is cited, in accordance with accepted academic practice. No use, distribution or reproduction is permitted which does not comply with these terms. 